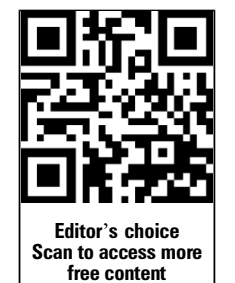

${ }^{1}$ Department of Pediatrics, Columbia University College of Physicians \& Surgeons, Children's Hospital of New York at New York Presbyterian, New York, New York, USA ${ }^{2}$ Heilbrunn Department of Population and Family Health, Columbia University Mailman School of Public Health, New York, New York, USA ${ }^{3}$ University of Sydney School of Medical Sciences, Sydney, New South Wales, Australia

${ }^{4}$ Australian Herpes

Management Forum, Westmead, Australia

\section{Correspondence to} Dr Marina Catallozzi, Departments of Pediatrics and Population and Family Health, Columbia University Medical Center, 622 W 168th St, VC4449F, New York, NY 10032 USA

mc2840@columbia.edu

Received 8 January 2013 Revised 22 April 2013 Accepted 29 April 2013 Published Online First 23 May 2013
To cite: Catallozzi $\mathrm{M}$, Ebel SC, Chávez NR, et al. Sex Transm Infect 2013;89:650-652.

\title{
Understanding perceptions of genital herpes disclosure through analysis of an online video contest
}

\author{
Marina Catallozzi, ${ }^{1,2}$ Sophia C Ebel, ${ }^{1}$ Noé R Chávez, ${ }^{1}$ Lee S Shearer, ${ }^{1}$ \\ Adrian Mindel, $^{3,4}$ Susan L Rosenthal ${ }^{1}$
}

\section{ABSTRACT}

Objective The aims of this study were to examine pre-existing videos in order to explore the motivation for, possible approaches to, and timing and context of disclosure of genital herpes infection as described by the lay public.

Methods A thematic content analysis was performed on 63 videos submitted to an Australian online contest sponsored by the Australian Herpes Management Forum and Novartis Pharmaceuticals designed to promote disclosure of genital herpes.

Results Videos either provided a motivation for disclosure of genital herpes or directed disclosure without an explicit rationale. Motivations included manageability of the disease or consistency with important values. Evaluation of strategies and logistics of disclosure revealed a variety of communication styles including direct and indirect. Disclosure settings included those that were private, semiprivate and public. Disclosure was portrayed in a variety of relationship types, and at different times within those relationships, with many videos demonstrating disclosure in connection with a romantic setting.

Conclusions Individuals with genital herpes are expected to disclose to susceptible partners. This analysis suggests that understanding lay perspectives on herpes disclosure to a partner may help healthcare providers develop counselling messages that decrease anxiety and foster disclosure to prevent transmission.

\section{INTRODUCTION}

Disclosure of herpes simplex viruses (HSV) to a partner has been shown to be protective against transmission. ${ }^{1}$ There is consensus that individuals should disclose to sexual partners ${ }^{2-4}$; however, individuals infected with genital herpes are often anxious about disclosing and worry about the stigma associated with having a sexually transmitted infection (STI). ${ }^{5} 6$ There has been limited research on patient or lay perspectives on disclosure, and understanding these perspectives may enhance counselling efforts. We analysed the motivation for, possible approaches to, and timing and context of, disclosure as portrayed in 30 -s videos submitted to an online public contest designed to promote disclosure.

\section{METHODS}

The Australian Herpes Management Forum (AHMF) and Novartis Pharmaceuticals challenged contestants to develop videos to communicate how you tell a partner you have HSV' in a 2011 on-line contest. Submissions were original films that were $30 \mathrm{~s}$ or shorter. A Judges' Choice Award (AUD $\$ 10000$ ) and five People's Choice Awards (AUD $\$ 1000$ ) were determined by contest organisers. The webpage is no longer active (http://www.liveandlove. com.au) and information about the filmmakers was not available. Specific information about the videos can be requested from the authors. An Institutional Review Board waiver was granted.

Of the 69 video submissions posted, 63 met criteria for analysis. Three aspects of disclosure (motivation, strategies and logistics, and timing and context within the relationship) were coded in table 1 . The analytic process was similar to the analysis performed on the 2010 contest videos focused on decreasing stigma. ${ }^{7}$ Coding focused on the process of disclosure rather than the outcome because most individuals evaluate outcome by the impact on the status of the relationship and most of the videos ended at the disclosure.

\section{RESULTS}

\section{Motivation for disclosure}

Videos demonstrating explicit rationales were classified as: disclose because HSV is manageable, disclose because it is consistent with important values (eg, honesty, love) and disclose to avoid being 'outed.' Videos sometimes included more than one motivation.

The manageability of the disease was the most common rationale. These videos typically focused on the ease of managing symptoms, the possibility of engaging in alternative sexual behaviours and the prevalence of herpes infections.

Values-based disclosure videos suggested that disclosure is the 'right' course of action in a relationship. They usually encouraged telling a partner because of love, respect or honesty.

A small number of videos directed individuals to disclose without discussing a rationale. The Judges' Choice Award video was an example of one that issued a direction. The first direction given was, "So, you have herpes. Well, you need to tell your partner." This video did not provide any rationale for disclosure; instead focused on each critical aspect of disclosure (eg, privacy).

\section{Strategies and logistics of disclosure}

Videos were coded according to whether the disclosure took place in private or not, was direct or 
Table 1 Coding scheme and frequencies

\begin{tabular}{|c|c|c|}
\hline & Sub-code & Number of videos $(n=63)$ \\
\hline Motivations for disclosure (not mutually exclusive) & $\begin{array}{l}\text { HSV is manageable } \\
\text { Disclosure is consistent with values (eg, honesty, love) } \\
\text { Directed/commanded without rationale } \\
\text { Shouldn't have secrets } \\
\text { To avoid being 'Outed' } \\
\text { No code }\end{array}$ & $\begin{array}{r}25 \\
14 \\
12 \\
4 \\
3 \\
17\end{array}$ \\
\hline Strategy and logistics of disclosure (not mutually exclusive) & $\begin{array}{l}\text { Direct communication } \\
\text { Private place } \\
\text { Indirect communication } \\
\text { Considerate of partner reaction } \\
\text { Recommends practicing disclosure } \\
\text { Recommends staying calm } \\
\text { No code }\end{array}$ & $\begin{array}{r}37 \\
33 \\
27 \\
6 \\
6 \\
2 \\
4\end{array}$ \\
\hline $\begin{array}{l}\text { Timing and context of disclosure (mutually exclusive) within } \\
\text { a relationship type (indiscriminate, just sexual, dating) }\end{array}$ & $\begin{array}{l}\text { Emphasis on before physical intimacy } \\
\text { Emphasis on as soon as diagnosed } \\
\text { Emphasis on after physical intimacy } \\
\text { In context of relationship, timing unclear } \\
\text { As the relationship progressed } \\
\text { No code }\end{array}$ & $\begin{array}{r}13 \\
10 \\
4 \\
21 \\
6 \\
9\end{array}$ \\
\hline
\end{tabular}

HSV, herpes simplex viruses.

indirect, was considerate of the partner's emotional reaction, was practiced or practice was suggested, and whether staying calm was advised. These categories were not mutually exclusive.

The majority of videos demonstrated or recommended disclosing in a private location, such as a home. In many of the 'private' settings, partners were shown in some romantic context. As noted earlier, it was not possible to code outcome; however, none of these videos indicated that this setting was problematic. There were some videos in which the private place was semiprivate, such as a café or park.

The most commonly demonstrated strategy was a direct communication with the partner, usually via in-person conversation, though three videos showed this communication occurring by phone or text message. In a video that portrayed disclosure through text messaging, a woman believes her partner is getting a high performance car (a Holden Special Vehicle, sometimes referred to as an HSV) and inadvertently shares the text, and his herpes diagnosis, with her friends. A smaller proportion of videos illustrated an indirect strategy. These included use of a game (eg, charades or board game), use of writing on the clothing or body, use of a pamphlet or information about herpes or leaving antiviral medication on the counter.

A few videos showed an awareness that the partner who is receiving the disclosure might need to have time and/or physical space to think about and integrate the information.

Other videos demonstrated or recommended practicing for the disclosure encounter in advance, including practicing with a friend, alone in the mirror or with an inanimate object like a teddy bear.

\section{Timing and context of disclosure}

Disclosure was shown in a variety of 'relationships' including indiscriminate disclosure without a relationship, a relationship that is just sexual or a dating relationship. Across these types of relationships, the timing of disclosure was coded as immediately after diagnosis, in the context of an existing relationship (where it was unclear if the diagnosis was new), before or after physical intimacy, or as the relationship progressed.

\section{Indiscriminate disclosure and just sexual relationship}

There were two videos that showed indiscriminate disclosure. The first showed a woman leaving an outgoing voicemail message that revealed her HSV status and the second showed a man disclosing to each potential partner during speed dating. These were both portrayed as examples of what should not be done. A minority of videos demonstrated disclosure in the context of a relationship that was portrayed as purely sexual.

\section{Within a dating relationship}

The majority of the videos demonstrated disclosure in the context of a clear dating relationship; however, the timing of the disclosure varied. It was sometimes clear that the disclosure was prompted by a new diagnosis. Other times, it was unclear. An example is a couple meeting in a café where the male partner has a litany of complaints about his day, including the fact that he has herpes. One cannot discern whether this diagnosis was pre-existing or if it was a new diagnosis that contributed to his bad day.

Timing around intimacy also varied within dating relationships. An example of disclosure prior to sexual intimacy shows a couple intimately kissing; the male stops to disclose his status, stating, "before we get to third base, oh I've got to reveal." Another video shows a man disclosing to his female partner postcoitally. Some videos specifically stated that if a relationship is "getting serious," disclosure is necessary.

\section{DISCUSSION}

Contest guidelines did not specify that a rationale for disclosure was required; however, most videos provided one, suggesting that providing rationales to individuals with HSV infection may be important as they decide to disclose to partners. Possibly because of the stigma associated with an STI, manageability and prevalence of the disease emerged as frequent motivations for disclosure. Healthcare providers (HCPs) could focus on these during counselling around disclosure to diminish the difficulties of disclosing genital infections. This may encourage disclosure and the notion that having HSV does not need to be a secret. ${ }^{8} 9$

While many resources promote direct in-person disclosure of HSV status, ${ }^{3}$ these videos contained a wide range of creative methods. Technology (phones, text messages) may be helpful if disclosure feels difficult to do in person. For patients who prefer this method, HCPs should review the potential risks of using technology, for example, the potential for 'viral spread.' Patient 
counselling can help the individual consider a variety of innovative strategies that would be effective and appropriate in terms of the characteristics of both partners and the relationship.

HCP counselling and patient literature suggest that disclosure should occur before any risk of exposure and not in the context of a physically intimate situation. ${ }^{3}{ }^{4}$ However, patients may find it difficult to determine how soon is 'too soon', and it has been demonstrated that many individuals feel it is less necessary to disclose in casual relationships. ${ }^{10}$ This struggle was evident in that some videos presented disclosure relative to increasing emotional commitment and others to increasing physical intimacy. Given that videos portrayed disclosure in intimate and romantic settings, HCPs should reconsider counselling messages that focus on avoiding disclosure in romantic/sexual situations. Counselling messages need to match with patient experiences and preferences.

This study was an analysis of videos created for an on-line contest and not for the purpose of this study. The videos were created in Australia; therefore, the results may not be generalisable to all cultures. The research team includes expertise in Australian culture including Australian perspectives on humour, sexuality and STIs.

Despite these limitations, this analysis revealed possible disconnects between current provider-based recommended counselling messages and the lay public's perceptions of needs including: a focus on explaining the ease of managing the disease as part of disclosure, using indirect and/or nonface-to-face strategies, and offering a variety of timings and settings. It is not possible to know whether these publically derived strategies are more effective, which approach would work best in which context, or how to provide guidance to patients concerned about domestic violence resulting from disclosure. Future research should focus on the relevant content regarding manageability, setting, method and timing of disclosing to partners as well as the partner experience and outcome. By collecting patient experiences in a rigorous scientific manner, we can develop recommendations that consider the needs of patients within specific relationships and decrease anxiety to foster timely disclosure.

\section{Key messages}

- Providing a motivation for disclosure and considering innovative methods (e.g., not direct conversation) may support the disclosure of genital herpes infection.

- Genital herpes disclosure may be occurring in a variety of settings including romantic ones and at a variety of times in relationships.

- Greater understanding is needed as to how to counsel patients in a manner that will reduce anxiety and promote disclosure of genital herpes.

\section{Handling editor Jackie A Cassell}

Acknowledgements The authors would like to acknowledge Charles Ebel for review of the paper and helpful suggestions. We also acknowledge Novartis Pharmaceuticals and the Australian Herpes Management Forum who organised the original contest from which the videos were obtained.

Contributors MC, SCE and SLR coded all the videos and wrote the manuscript. NRC and LSS helped to develop the thematic coding and provided comments on the interpretation of the data and writing of the manuscript. AM was involved in the original creation of the video contest, provided information about the Australian context and insights about the relevance of the findings for STI clinicians, and provided comments on the interpretation and writing.

Competing interests None.

Provenance and peer review Not commissioned; externally peer reviewed.

\section{REFERENCES}

1 Wald A, Krantz E, Selke $S$, et al. Knowledge of partners' genital herpes protects against herpes simplex virus type 2 acquisition. J Infect Dis 2006;194:42-52.

2 Morbidity and Mortality Weekly Report. Sexually Transmitted Diseases Treatment Guidelines, 2010. http://www.cdc.gov/mmwr/preview/mmwrhtml/rr5912a1.htm. 17 December 2010 (accessed 29 Nov 2012).

3 Ebel C, Wald A. Managing herpes: how to live and love with a chronic STD. ResearchTriangle Park: American Social Health Association, 2002:121-46.

4 Warren T. The good news about the bad news: herpes: everything you need to know. Oakland, CA: New Harbinger Publications, 2009:107-31.

5 Patrick DM, Rosenthal SL, Stanberry LR, et al. Patient satisfaction with care for genital herpes: Insights from a global survey. Sex Transm Infect 2004;80:192-7.

6 Merin A, Pachankis JE. The psychological impact of genital herpes stigma. J Health Psychol 2011;16:80-90.

7 Shearer LS, Simmons L, Mindel A, et al. Reducing the stigma of herpes simplex virus infection: Lessons from an on-line video contest. Sex Health 2012;9:438-44.

8 Newton DC, McCabe M. Effects of sexually transmitted infection status, relationship status, and disclosure status on sexual self-concept. J Sex Res 2008;45:187-92.

9 Bickford J, Barton SE, Mandalia S. Chronic genital herpes and disclosure.... The influence of stigma. Int J STD AIDS 2007;18:589-92.

10 Green J, Ferrier $S$, Kocsis $A$, et al. Determinants of disclosure of genital herpes to partners. Sex Transm Infect 2003;79:42-4. 